\title{
Educação em saúde e prevenção do câncer de mama no município de Itaúna, Minas Gerais
}

RESUMO | Objetivo: Relatar a experiência de ações educativas realizadas com profissionais da Estratégia de Saúde da Família, sobre a prevenção do câncer de mama e o incentivo ao autoexame. Métodos: Trata- se de um estudo descritivo, do tipo relato de experiência, realizado com 28 profissionais da saúde no município de Itaúna, Minas Gerais, entre setembro à outubro de 2019. Foi utilizado um questionário de pré e pós-intervenção para comparar conhecimentos prévios e adquiridos, além de uma atividade prática de palpação em próteses de mamas. Resultados: Houve predominância de participantes com idade entre 31 a 38 anos (35,71\%), seguida de profissionais entre 23 a 30 anos (32,14\%). Os resultados mostraram que $60,71 \%$ dos participantes relataram ter conhecimento sobre o assunto, 28,57\% afirmaram não saber do que se trata e 10,72\% não responderam a essa questão. Conclusão: A intervenção aplicada serve de base para que estes profissionais disseminem os novos saberes para população.

Palavras-chaves: Agentes comunitários de saúde; Educação em saúde; Neoplasias da mama; Técnicos de enfermagem.

ABSTRACT | Objective: To report the experience of educational actions carried out with professionals from the Family Health Strategy, on the prevention of breast cancer and the encouragement of self-examination. Methods: This is a descriptive, experience report type study conducted with 28 health professionals in the city of Itaúna, Minas Gerais, between September and October 2019. A pre- and post-intervention questionnaire was used to compare knowledge and acquired, in addition to a practical palpation activity on breast prostheses. Results: There was a predominance of participants aged between 31 and 38 years old (35.71\%), followed by professionals between 23 and 30 years old (32.14\%). The results showed that $60.71 \%$ of the participants reported having knowledge about the subject, $28.57 \%$ said they did not know what it was about and $10.72 \%$ did not answer this question. Conclusion: The applied intervention serves as a basis for these professionals to disseminate new knowledge to the population.

Keywords: Community health workers; Health education; Breast neoplasms; Nursing technicians.

RESUMEN | Objetivo: Informar sobre la experiencia de las acciones educativas llevadas a cabo con profesionales de la Estrategia de Salud de la Familia, sobre la prevención del cáncer de mama y el fomento del autoexamen. Métodos: Este es un estudio descriptivo tipo informe de experiencia realizado con 28 profesionales de la salud en la ciudad de Itaúna, Minas Gerais, entre septiembre y octubre de 2019. Se utilizó un cuestionario previo y posterior a la intervención para comparar el conocimiento. y adquirido, además de una práctica actividad de palpación en prótesis mamarias. Resultados: predominaron los participantes de edades comprendidas entre 31 y 38 años (35,71\%), seguidos por profesionales de entre 23 y 30 años (32,14\%). Los resultados mostraron que el $60.71 \%$ de los participantes informaron tener conocimiento sobre el tema, el $28.57 \%$ dijo que no sabían de qué se trataba y el $10.72 \%$ no respondió a esta pregunta. Conclusión: La intervención aplicada sirve como base para que estos profesionales difundan nuevos conocimientos a la población.

Palavras claves: Trabajadores comunitarios de salud; Educación para la salud; Neoplasias de mama; Técnicos de enfermería.

Greice Kelly de Oliveira Alves

Enfermeira. Graduação em Enfermagem pela Universidade de Itaúna (UIT), Minas Gerais. https://orcid.org/0000-0002-5868-9993

\section{Geisiane Aparecida da Silva}

Graduanda em Enfermagem pela Universidade de Itaúna (UIT), Minas Gerais. https://orcid.org/0000-0003-2377-9953

\section{Marla Ariana Silva}

Graduanda em Enfermagem pela Universidade do Estado de Minas Gerais (UEMG), Unidade Divinópolis. https://orcid. org/0000-0003-0136-7122

\section{Karen dos Santos Lago}

Graduanda em Enfermagem pela Universidade do Estado de Minas Gerais (UEMG), Unidade Divinópolis.https://orcid. org/0000-0002-4502-5098

\section{Silmara Nunes Andrade}

Enfermeira. Doutora em Ciências da Saúde pela Universidade Federal de São João Del Rei (UFSJ). Docente do Curso de Graduação em Enfermagem da Universidade do Estado de Minas Gerais (UEMG), Unidade Divinópolis. https://orcid.org/0000-0002-1975-0827

\section{Regina Consolação dos Santos}

Enfermeira. Mestre em Ciência pela Universidade Federal de São João Del Rei, (UFSJ). Docente e Coordenadora do Curso de Graduação em Enfermagem da Universidade de Itaúna (UIT). Docente do Curso de Graduação em Enfermagem da Universidade do Estado de Minas Gerais (UEMG), Unidade Divinópolis. https://orcid. org/0000-0002-7393-3210

Recebido em: 16/07/2020

Aprovado em: 20/07/2020
INTRODUÇÃO

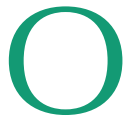
câncer de mama configura-se como um dos desafios do envelhecimento populacional, e das doenças crônicas que assolam esse processo. É o tipo de câncer que mais acomete e mata mulheres no Brasil, excetuando-se os tumores de pele não melanoma $^{(1)}$. É considerado problema de saúde pública, devido a dificuldade na garantia do acesso integral da população ao diagnóstico e tratamento desta doença pelo sistema de saúde ${ }^{(2)}$.

A neoplasia maligna da mama, como também é conhecida, é resultado da multiplicação desordenada de células da mama que podem invadir outros tecidos. Alguns tumores podem desenvolver- se mais rápido que outros, contudo 
a maioria dos casos tem bom prognóstico perante um diagnóstico precoce e o tratamento. Os tumores podem ser detectados em diferentes fases (estadiamento), sendo in situ os localizados em apenas um local, e infiltrantes quando invadem outros órgãos ou atingem linfonodos ${ }^{(3)}$.

De acordo com estatísticas mundiais recentes do Globocan 2018, foi estabelecida uma incidência de 2,1 milhões de casos de câncer e 627 mil mortes pela doença $^{(4)}$. Em 2016 no Brasil, houve 16.069 óbitos de mulheres pelo CA de mama ${ }^{(1)}$.

Estimativas para cada ano do biênio de 2018-2019 ressaltam que no Brasil são esperados 59.700 casos novos de câncer de mama, com um risco presumido de 56,330 casos a cada 100 mil mulheres. Estima-se que no período de 2018-2019, na região sudeste, o número de casos incidentes por ano seja de aproximadamente $30.880^{(5)}$.

É importante ressaltar que apesar de não possuir uma etiologia única, alguns fatores de risco predispõe a manifestação da doença. Ser mulher e a idade continuam sendo os fatores mais importantes, com uma incidência maior até os 50 anos. Além dos fatores relacionados a vida reprodutiva da mulher como, menarca precoce e menopausa tardia, não ter tido filhos ou gravidez após 30 anos, não ter amamentado, uso de anticoncepcional oral e terapia de reposição hormonal pós- menopausa. Outros fenômenos que podem contribuir para o desenvolvimento do câncer estão relacionados à alterações genéticas, história familiar com a doença e alta densidade das mamas ${ }^{(6,1)}$.

No que se refere aos fatores ambientais, estudos indicam o sobrepeso, sedentarismo, tabagismo, consumo abusivo de bebida alcoólica e exposição frequente à radiação. Nessa conjuntura, pode- se afirmar que o controle dos elementos supracitados fomentaria no retardo ou até mesmo na prevenção dessa neoplasia em um terço da população acometida ${ }^{(6)}$.

Em consonância, a promoção de atividades de educação em saúde na Atenção Básica por meio da disseminação de

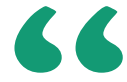

Estimativas para cada ano do biênio de 2018-2019 ressaltam que no Brasil são esperados 59.700 casos novos de câncer de mama, com um risco presumido de 56,330 casos a cada 100 mil mulheres. Estimase que no período de 2018-2019, na região sudeste, o número de casos incidentes por ano seja de aproximadamente 30.880 . conhecimentos acerca do CA de mama e seus fatores de risco, promovendo a participação ativa da população e incentivando o diagnóstico precoce, são de grande relevância para adesão de hábitos de vida saudáveis visando uma mudança no atual cenário em que se encontra a doença ${ }^{(6)}$.

A Atenção Primária a Saúde (APS) caracteriza-se como o principal local para o desenvolvimento dessas ações, pois além de utilizar o modelo de Estratégia de Saúde da Família (ESF) é porta de entrada prioritária do SUS, com a finalidade de integrar e resolver a maioria dos problemas da população ${ }^{(7)}$. Nesse contexto, destaca-se a relação profissional- usuário presente nesse nível de atenção, que proporciona um vínculo sem que a mulher possua alguma patologia (ou não), estabelecendo uma relação de confiança mútua e possibilitando o protagonismo dessa mulher em seu autocuidado ${ }^{(2)}$.

A atuação do enfermeiro na APS para detecção precoce do CA de mama é essencial, incluindo ações de promoção à saúde, tratamento e reabilitação. Destacam- se como funções do enfermeiro no controle do CA de mama a realização de consultas com Exame Clínico das Mamas (ECM) de acordo com a idade e quadro clínico, observar sinais e sintomas referentes a neoplasia, solicitar e analisar exames, referenciar para os serviços especializados e acompanhar a evolução do processo de diagnóstico e/ ou tratamento, desenvolver e participar de atividades de educação permanente. Contudo, pesquisas recentes demonstram a necessidade de capacitação desses profissionais em relação ao tema, devido ao escasso entendimento acerca dos fatores de risco, métodos de triagem e insuficiente educação continuada, o déficit de tais conhecimentos pode interferir no plano de intervenções proposto pelo Ministério da Saúde para controle da doença ${ }^{(7)}$. Justificando assim a implementação deste projeto de pesquisa.

Objetivou-se relatar a experiência de ações educativas realizadas com profissionais da Estratégia de Saúde da Família 
sobre a prevenção do câncer de mama e o incentivo ao autoexame.

\section{MÉTODOS}

Trata- se de um estudo descritivo, do tipo relato de experiência, relacionado à ação educativa em saúde com profissionais de ESF, referente ao câncer de mama. O projeto realizado por graduandos de enfermagem da Universidade de Itaúna (UIT) promoveu capacitação acerca do câncer de mama, seus fatores de risco e prevenção, além do incentivo ao autoexame das mamas, através de palestras e mesas redondas com auxílio de recursos visuais, de palpação e avaliação de conhecimentos sobre o assunto.

O Município de Itaúna localiza-se na região Centro-Oeste do estado de Minas Gerais, e segundo estimativas do instituto Brasileiro de Geografia e Estatística, o município possui aproximadamente 93.214 habitantes ${ }^{(8)}$. Segundo dados da Secretaria Municipal de Saúde de Itaúna, o município possui 22 unidades de saúde na rede de Atenção Primária à Saúde (APS), sendo 20 ESF urbanas, 01 ESF de zona rural (sendo esta itinerante) e 01 Unidade de Saúde no modelo de atendimento tradicional ${ }^{(9)}$.

O público alvo foi composto por um total de 28 participantes, entre eles agentes comunitários, técnicos de enfermagem e outros profissionais inseridos nas ESF's, vinculada à Secretaria de Saúde do município de Itaúna, Minas Gerais. Para o recrutamento dos participantes foram delimitados os seguintes critérios de inclusão: ser maior de 18 anos, estar em regime de trabalho efetivo (concursado) ou contrato (temporário) e estar lotados nas ESF's do município no momento da coleta. Os critérios de exclusão foram: os profissionais que estavam afastados de suas atividades laborais durante o período de coleta de dados.

Para este estudo foram escolhidas 05 unidades por conveniência: facilidade de acesso, facilidade da realização das visitas, menor custo e maior viabilização do
O projeto realizado por graduandos de enfermagem da Universidade de Itaúna (UIT) promoveu capacitação acerca do câncer de mama, seus fatores de risco e prevenção, além do incentivo ao autoexame das mamas, através de palestras e mesas redondas com auxílio de recursos visuais, de palpação e avaliação de conhecimentos sobre o assunto. presente trabalho. As ações aconteceram entre os meses de setembro e outubro do ano de 2019, com duração média de 40 minutos por unidade, as mesmas eram previamente marcadas com as unidades em horário disponível para os profissionais e palestrante.

Em relação aos recursos utilizados, através de pesquisa literária do tema, foi criada apresentação em power point e posteriormente em banner, para auxiliar nas palestras. A mamamiga que foi fornecida pela Universidade, é composta por três próteses de mama, na qual uma delas não apresenta alterações e as demais possuem nódulos palpáveis e deformação na pele, contribuindo para a prática de palpação e instrução do autoexame das mamas.

A ação foi divida em três momentos, sendo o primeiro da pré-avaliação, o segundo de exposição do conteúdo, esse foi constituído pela definição da neoplasia mamária e alguns dados epidemiológicos da doença, estrutura básica das mamas, fases e tipos de câncer de mama (in situ e infiltrante/ ductal e lobular), fatores de risco, sintomas, detecção precoce com o rastreamento como estratégia e medidas para prevenção como o autoexame das mamas. Por último os ouvintes eram incentivados a trocar experiências, esclarecer dúvidas e apalpar a mamamiga, enquanto respondiam ao questionário de pós-informações.

A partir da compreensão do tema proposto, foi aplicado um questionário para avaliação dos conhecimentos prévios e efetividade da palestra, constituído por 06 assertivas sobre o câncer de mama em geral e 09 possíveis fatores de risco, no qual os mesmos deveriam ser marcados apenas com "sim ou não", antes e depois da exposição dos conteúdos. O objetivo era comparar as informações que os participantes da pesquisa dispunham antes da intervenção, e os adquiridos após a atividade educativa. Em seguida os dados empíricos coletados foram tratados com estatística simples e registrados no software Microsoft Office Excel (2010) que contemplou a estruturação de um banco de dados. 
Vale ressaltar que não houve necessidade de apreciação por parte do Comitê de Ética em Pesquisa (CEP)/Comissão Nacional de Ética em Pesquisa (CONEP), por se tratar de um relato de experiência, mas o mesmo atendeu os princípios éticos conforme resolução do Conselho Nacional de Saúde 466/2012 ${ }^{(10)}$. Todos os participantes assinaram o Termo de Consentimento Livre e Esclarecido (TCLE) antes do início das entrevistas.

\section{RESULTADOS}

No primeiro momento do questionário foram caracterizados os participantes do estudo (nome, idade, sexo, profissão). No segundo momento os participantes foram orientados a responderem um questionário com dois mediadores a respeito do assunto câncer de mama e seus fatores de risco, para que em um terceiro momento, fosse realizada a ação de expansão de saberes a partir de um raciocínio científico

\section{Tabela 1- Conhecimentos acerca do câncer de mama.}

\begin{tabular}{|c|c|c|c|c|c|c|c|c|}
\hline \multirow[b]{3}{*}{ Câncer de mama é: } & \multicolumn{4}{|c|}{ Antes } & \multicolumn{4}{|c|}{ Depois } \\
\hline & \multicolumn{2}{|c|}{ Sim } & \multicolumn{2}{|c|}{ Não } & \multicolumn{2}{|c|}{ Sim } & \multicolumn{2}{|c|}{ Não } \\
\hline & $\mathrm{N}^{\circ}$ & $\%$ & $\mathrm{~N}^{\circ}$ & $\%$ & $\mathrm{~N}^{\circ}$ & $\%$ & $\mathrm{~N}^{\circ}$ & $\%$ \\
\hline Doença que não tem cura & 3 & 10,71 & 25 & 89,29 & 5 & 17,86 & 23 & 82,14 \\
\hline Doença sem prevenção & 5 & 17,86 & 23 & 82,14 & 3 & 10,71 & 25 & 89,29 \\
\hline Doença genética & 27 & 96,43 & 1 & 3,57 & 27 & 96,43 & 1 & 3,57 \\
\hline Doença que tem cura & 27 & 96,43 & 1 & 3,57 & 26 & 92,86 & 2 & 7,14 \\
\hline Doença de pessoas idosas & 5 & 17,86 & 23 & 82,14 & 4 & 14,29 & 24 & 85,71 \\
\hline Não sabe nada a respeito & 8 & 28,57 & 17 & 60,71 & 5 & 17,86 & 20 & 71,43 \\
\hline \multicolumn{9}{|l|}{ Fatores de risco } \\
\hline Sobrepeso & 20 & 71,43 & 8 & 28,57 & 27 & 96,43 & 1 & 3,57 \\
\hline Sedentarismo & 22 & 78,57 & 6 & 21,43 & 28 & 100 & 0 & 0 \\
\hline Tabagismo & 22 & 78,57 & 6 & 21,43 & 28 & 100 & 0 & 0 \\
\hline Anticoncepcional & 17 & 60,71 & 11 & 39,29 & 27 & 96,43 & 1 & 3,57 \\
\hline Bebida alcoólica & 17 & 60,71 & 10 & 35,71 & 27 & 96,43 & 1 & 3,57 \\
\hline Radiação & 21 & 75 & 7 & 25 & 27 & 96,43 & 1 & 3,57 \\
\hline Não ter filhos & 6 & 21,43 & 22 & 78,57 & 24 & 85,71 & 3 & 10,71 \\
\hline Amamentação prolongada & 3 & 10,71 & 25 & 89,29 & 2 & 7,14 & 24 & 85,71 \\
\hline Histórico na família & 26 & 92,86 & 2 & 7,14 & 25 & 89,29 & 1 & 3,57 \\
\hline
\end{tabular}

Fonte: Pesquisa de campo, 2020 e prático sobre o tema. Na fase final, os ouvintes foram incentivados a trocar experiências e reconstruir suas respostas, baseadas nas informações adquiridas.

Os dados coletados em relação à idade dos participantes do estudo revelaram que houve predominância de participantes com idade entre 31 e 38 anos $(35,71 \%)$, seguida de profissionais entre 23 e 30 anos $(32,14 \%)$.

De acordo com o nível de conhecimento dos participantes sobre o autoexame de mamas, coletado no segundo momento da pesquisa, observou-se que $60,71 \%$ dos participantes relataram ter conhecimento sobre o assunto, 28,57\% afirmaram não saber do que se trata e $10,72 \%$ não responderam a essa questão.

$\mathrm{Na}$ tabela 1, apresentam-se dados comparativos relacionados aos conhecimentos acerca do câncer de mama, apontados antes e depois da ação realizada com os profissionais de saúde.

\section{DISCUSSÃO}

Diante disso, se faz necessário que sejam promovidas mais ações educativas para esses profissionais incentivando a atualização contínua em saúde. A chance de cura em decorrência de uma descoberta no início é de uma a cada três casos de câncer de mama. Mas o receio e a falta de conhecimento faz com que os pacientes não falem do assunto, o que acarreta em um atraso do diagnóstico. Por isso, é importante desmistificar a doença como uma sentença de morte inevitável ${ }^{(11)}$.

A compreensão dos critérios para desenvolvimento do câncer de mama foi insuficiente na avaliação pré-erudição, mas satisfatórios após intervenção, principalmente os fatores anticoncepcional, bebida alcoólica e não ter filhos. Já é sabido que mulheres em uso de contraceptivos hormonais, por longo tempo e antes da primeira gestação, constituem o principal grupo para desenvolvimento da neoplasia mamária ${ }^{(6)}$.

Em relação ao nível de ingestão de bebidas alcoólicas, o risco é proporcional ao tempo de exposição e a quantidade ingerida, é fator de predisposição para diversos tipos de câncer incluindo o de mama. $\mathrm{O}$ álcool libera radicais livres que podem causar danos ao DNA, e além de aumentar os níveis de estrogênio também atua como um solvente, facilitando a entrada de carcinógenos dietéticos ou ambientais nas células. $\mathrm{O}$ alto consumo desta substância ainda pode levar a insuficiência de nutrientes essenciais, tornando o tecido mamário mais vulnerável ${ }^{(1)}$.

O tema que mais teve modificação nas respostas após a palestra está associado à gestação. A maior parte dos participantes desta ação desconhecia que, não ter filhos era um fator de risco para o desenvolvimento do câncer de mama, no entanto sabiam que a amamentação prolongada não era uma ameaça. As duas asserções estão diretamente ligadas, uma vez que durante o período da gravidez e da amamentação ocorre uma redução dos hormônios que potencializam o risco para o câncer, além disso, o ato de amamentar promove a eli- 
minação de células mamárias com possível mutação. Por isso, quanto maior for a duração do aleitamento materno, melhores serão os benefícios, tanto para mãe quanto para o bebê $\hat{~}^{(1)}$.

Por conseguinte, o desconhecimento dos fatores de risco prejudica a promoção de ações para prevenção de doenças. Apesar de não ser possível intervir em todos, é de alta relevância que todos os profissionais de saúde saibam informar a população sobre os riscos aos quais estão susceptíveis.

Procedeu-se a ação com instruções para realização do autoexame das mamas, dentre estes: a inspeção no espelho e os critérios de periodicidade. Houve a demonstração, em prótese mamária, da direção dos movimentos com a finalização na expressão mamilar, e foi referido sobre a importância de se apalpar também as regiões da axila, supra e subclavicular, devido aos gânglios linfáticos ali presentes. Posteriormente deu- se a dinâmica de palpação pelos intervenientes.

Durante a dinâmica os participantes demonstraram interesse e curiosidade na atividade. A maioria revelou que não tinha o hábito de realizar o autoexame, apesar de saberem a respeito. A realização do autoexame é uma prática de autocuidado em que se estimula a responsabilidade do indivíduo com a saúde e a detecção do CA de mama ${ }^{(12)}$. A mídia está sempre divulgando a importância desta ação, porém o modo correto de realizá-la ainda é desconhecido pela população geral ${ }^{(13)}$.

Perante essa realidade, é imperativo que os serviços de saúde invistam mais nessa capacitação dos seus profissionais. Pois o autoexame é uma importante estratégia que visa o autoconhecimento da mulher em relação ao seu corpo, tonando-a responsável pelo seu cuidado além de proporcionar a oportunidade de um diagnóstico precoce, que aumenta as chances de cura no caso do câncer de mama.

No final da ação os profissionais foram fomentados a disseminar os conhecimentos adquiridos para os seus usuários, familiares e amigos, através de uma ação positiva e de conscientização sobre o câncer de mama, e principalmente a prevenção.

O presente estudo apresenta limitações, como o nível de escolaridade, a população em foco eram os agentes comunitários e técnicos de enfermagem (nível médio). Algumas respostas foram inapropriadas, pois determinava duas proposições opostas como corretas, outras foram incompletas (não foram marcadas). Durante toda a ação foi possível notar dificuldade na compreensão do questionário, por alguns profissionais, outros não se atentaram as explicações de como deveria ser preenchido. Porém, os resultados foram satisfatórios e o objetivo proposto foi atendido.

\section{CONCLUSÃO}

O atual cenário epidemiológico, no qual se encontra a situação em saúde do Brasil, exige atualização contínua dos seus profissionais não só do nível médio, mas também do nível superior. Sendo assim, é necessário que os gestores da rede pública de saúde invistam mais em capacitações aos seus funcionários, e implantem projetos como este para a população em geral, como forma prática de estabelecimento da saúde e prevenção de doenças. Em relação aos participantes, a intervenção aplicada serve de base para que estes profissionais disseminem os novos saberes para população.

\section{Referências}

1. Instituto nacional de câncer (INCA). A situação do câncer de mama no Brasil: síntese de dados dos sistemas de informação. Rio de Janeiro: INCA; 2019. Disponível em: https://www.inca.gov.br/sites/ufu.sti.inca.local/files// media/document//a_situacao_ca_mama_brasil_2019.pdf. Acesso em 09 Out. 2019.

2. Zapponi ALB, Tocantins FR, Vargens OMC. 0 enfermeiro na detecção precoce do câncer de mama no âmbito da atenção primária. Rev. enferm. UERJ, v.23, n.1, p. 33-38, jan.-fev. Rio de Janeiro; 2015.

3. Instituto Nacional de Câncer (INCA). A mulher e o câncer de mama no Brasil: uma realização do projeto "História do Câncer - atores, cenários e políticas públicas", parceria INCA-COC/Fiocruz. Ed. 3. Rio de Janeiro; 2018. Disponível em: https://www.inca.gov.br/publicacoes/apresentacoes/mulher-e-o-cancer-de-mama-no-brasil-exposicao-versao-em-pdf. Acesso em 09 Out. 2019

4. Bray F, et al. Global Cancer Statistics 2018: GLOBOCAN Estimates of Incidence and Mortality Worldwide for 36 Cancers in 185 Countries. CA: a Cancer Journal for Clinicians, v. 68, n. 6, p. 394-424, 2018.

5. Instituto Nacional De Câncer (INCA). Estimativa 2018: Incidência de câncer no Brasil. 2017. Disponível em: http://www.inca.gov.br/estimativa/2016/. 6. Bushatsky M, Cabral LR, Cabral JR, Barros MBS C, Gomes BMR, Figueira Filho ASS . Educação em saúde: uma estratégia de intervenção frente ao câncer de mama. Rev. Cienc Cuid Saúde, v. 14, n. 1, p. 870-878, jan.- mar. Recife; 2015.

7. Teixeira MS, Goldman RE, Gonçalves VCS, Gutiérrez MGR, Figueiredo
EN. Atuação do enfermeiro da Atenção Primária no controle do câncer de mama. Acta Paulista de Enfermagem, vol. 30, núm. 1, p. 1-7, jan.-fev. 2017. 8. Instituto brasileiro de geografia e estatística (IBGE). Estimativa da população 2019. Disponível em: https://cidades.ibge.gov.br/brasil/mg/itauna/ panorama. Acesso em: 10 Nov. 2019.

9. Prefeitura municipal de itaúna/mg. Secretaria Municipal de Saúde, 2018. Disponivel em: https://www.itauna.mg.gov.br/portal/secretarias/31/secretaria-municipal-de-saúde. Acesso em: 09 Out. 2019.

10. Brasil. Resolução $n^{\circ} 466$, de 12 de dezembro de 2012. Conselho Nacional de Ética em Pesquisa. Brasília, 2012. Disponível em: http://conselho. saude.gov.br/resolucoes/2012/Reso466.pdf. Acesso em: 18 Jul. 2020.

11. Instituto Nacional de Câncer (INCA). Câncer de mama: é preciso falar disso. Ed. 1. Rio de Janeiro; 2014. Disponível em: http://www.inca.gov.br. Acesso em: 09 Out. 2019.

12. Silva SR, Lício FC, Borges LV, Mendes LC, Vicente NG, Gomes NS. Atividades educativas na área da saúde da mulher: um relato de experiência. Revista de Enfermagem e Atenção à Saúde, v. 1, núm. 1, p. 107-112, out. - nov. 2012. Universidade Federal do triângulo Mineiro. Minas Gerais, Brasil. Disponível em: http://seer.uftm.edu.br/revistaeletronica/index.php/enfer/ article/view/299/283. Acesso em 06 Nov. 2019.

13. Jurado SR, Saraiva KVO, Wheis M, Pereira LVRC. Sintomas depressivos em mulheres com câncer de mama submetidas à quimioterapia e radioterapia: uma revisão integrativa. Revista Nursing, p. 2967-2972, 2019. Disponível em: https://pesquisa.bvsalud.org/portal/resource/pt/biblio-1025617. 